\title{
Stability of systems of three arbitrary charges: General properties
}

\section{Citation}

Martin, André, Jean-Marc Richard, and Tai Tsun Wu. 1995. "Stability of Systems of Three Arbitrary Charges: General Properties." Physical Review A 52 (4): 2557-63. https:// doi.org/10.1103/physreva.52.2557.

\section{Permanent link}

http://nrs.harvard.edu/urn-3:HUL.InstRepos:41555810

\section{Terms of Use}

This article was downloaded from Harvard University's DASH repository, and is made available under the terms and conditions applicable to Other Posted Material, as set forth at http:// nrs.harvard.edu/urn-3:HUL.InstRepos:dash.current.terms-of-use\#LAA

\section{Share Your Story}

The Harvard community has made this article openly available.

Please share how this access benefits you. Submit a story.

Accessibility 


\title{
Stability of systems of three arbitrary charges: General properties
}

\author{
André Martin \\ CERN, Theory Division, CH-1211 Geǹeve 23, Switzerland \\ Jean-Marc Richard \\ Institut des Sciences Nucléaires CNRS-IN2P3, Université Joseph Fourier, Grenoble Cédex, France \\ and European Centre for Theoretical Studies in Nuclear Physics and Related Areas, University of Trento, Trento, Italy \\ Tai Tsun Wu \\ Gordon McKay Laboratory, Harvard University, Cambridge, Massachussets 02138 \\ and CERN, Theory Division, $\mathrm{CH}-1211$ Gerieve 23, Switzerland \\ (Received 31 May 1994; revised manuscript received 1 March 1995)
}

\begin{abstract}
The stability of systems consisting of a negative charge $-q_{1}$ and two positive charges $q_{2}$ and $q_{3}$ is discussed as a function of the values of the charges $q_{i}$ and of the constituent masses $m_{i}$. We give general results and list some open questions of interest, which hopefully will stimulate further investigations.
\end{abstract}

PACS number(s): 03.65.Ge, 31.15.- $\mathrm{p}, 36.10 .-\mathrm{k}$

\section{INTRODUCTION}

Recently, several authors have reviewed the problem of the stability of systems made of three unit charges, say $Q_{i}= \pm(-1,+1,+1)[1-4]$. This includes some wellknown cases such as the ionized hydrogen molecule $\mathrm{H}_{2}{ }^{+}$ $\left(e^{-} p p\right)$, the hydrogen ion $\mathrm{H}^{-}\left(p e^{-} e^{-}\right)$, or the positronium ion $\left(e^{+} e^{-} e^{-}\right)$, which are stable, and the positronhydrogen system $\left(e^{-} p e^{+}\right)$which is unbound. The question is for which constituent masses $m_{i}$ the system remains stable against dissociation into an atom and an isolated charge.

A natural extension consists of allowing the charges $Q_{i}$ to vary, i.e., considering a system with

$$
Q_{i}= \pm\left(-q_{1}, q_{2}, q_{3}\right), \quad q_{i}>0,
$$

and studying the stability domain as a function of the variables $q_{i}$. The practical applications are limited, at first sight. However, there are systems in nature like the proton, $\alpha$ particle, and electron for which one can ask the question of stability (in this particular case the answer is negative according to Chen and Spruch [5]). We hope, however, to understand more deeply the mechanisms by which collective binding sometimes becomes more favorable than splitting into small clusters.

In the course of our investigations, we have soon checked our expectation that this problem is extremely complicated. The system is governed by four parameters, two mass ratios and two charge ratios, and in most cases deciding the stability would require performing an accurate numerical calculation. We would like to stress that here we restrict ourselves to the problem of strict stability, i.e., stability of the ground state. For some systems, like proton-muon- $\alpha$-particle, one can speak of "quasistability" of certain excited states according to the terminology of Gershtein and Ponomarev [6], i.e., stability in the limit where the masses of the nuclei become very large.
Our paper will therefore not display many concrete results. It will instead focus on selected open questions. Hopefully this will stimulate further developments on the rigorous properties of the stability domain, and perhaps some new numerical investigations.

The paper is organized as follows. In Sec. II, we briefly summarize the results obtained for unit charges $q_{i}=1$. The simple properties of the stability domain for arbitrary charges are explained in Sec. III. In Sec. IV, we discuss the case where the positive charges are infinitely massive.

In Sec. $\mathrm{V}$, we consider the case of nearly symmetric systems, with equal masses $m_{2}=m_{3}$, and neighboring charges $q_{2} \simeq q_{3}$. The possible shapes of the stability domain in the plane of inverse masses are presented in Sec. VI, while in Sec. VII we display the complementary slices of the domain: the stability as a function of the charges, for given masses. In the conclusions (Sec. VIII), we stress some of the most challenging open questions.

In a forthcoming paper [7], we shall study in more detail some critical cases which require specific developments, for instance, the case of small $q_{3}$.

\section{RESULTS FOR UNIT CHARGES}

In Ref. [1], we studied the properties of the stability domain for the case of three unit charges, i.e., $q_{i}=1$ in

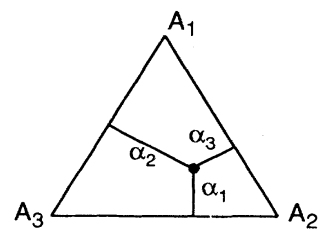

FIG. 1. Domain of possible inverse masses $\alpha_{i}$ submitted to $\sum \alpha_{i}=1$. 


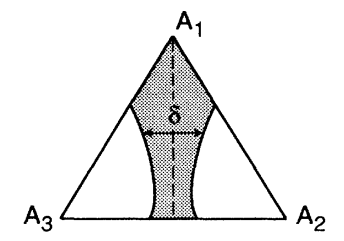

FIG. 2. Schematic shape of the stability domain in the case of equal charges $q_{i}=1$.

our notation. A comprehensive review with many references is given in [4].

We first stressed that the inverse masses $\alpha_{i} \equiv 1 / m_{i}$ are better variables than the masses $m_{i}$ themselves to account for the regularities of the binding energy when one changes the constituents without modifying the potential. Using the scaling properties of the Coulomb interaction, we can impose, without loss of generality,

$$
\sum_{i=1}^{3} \alpha_{i}=1
$$

so that all possible cases can be represented by the interior of an equilateral triangle, as shown in Fig. 1. The $\alpha_{i}$ are the barycentric coordinates in this plot.

The stability region is displayed in Fig. 2. It consists of a rather narrow band around the symmetry axis $\alpha_{2}=\alpha_{3}$ where like charges have the same mass. The shape of the frontier results from the three basic properties.

(i) Each instability region is star shaped with respect to the vertex of the triangle which it includes. This means that a straight line issuing from $A_{2}$ in Fig. 2 will cross at most once the stability frontier between $A_{2}$ and the vertical axis.

(ii) Each instability region is convex, i.e., if two points belong to the domain the whole segment connecting the two points belongs to the domain.

(iii) Every symmetric configuration $\left(m_{2}=m_{3}\right)$ is stable.

The proof of (iii) was given by Hill [8]. The proof of (i) and (ii) essentially relies on the binding energy being an increasing and concave function of each $\alpha_{i}$, as explained in Ref. [1].

\section{GENERAL RESULTS ON THREE-CHARGE SYSTEMS}

Using the definition (1) of the charges, we write the three-body Hamiltonian as

$$
H=\sum_{i} \frac{\alpha_{i}}{2} \vec{p}_{i}^{2}+\frac{q_{2} q_{3}}{r_{23}}-\frac{q_{1} q_{2}}{r_{12}}-\frac{q_{1} q_{3}}{r_{13}},
$$

with $r_{i j}-\left|\vec{r}_{i}-\vec{r}_{j}\right|$. It has the following properties.

\section{A. Scaling}

We already mentioned the elementary scaling properties of the Coulomb interaction. A simultaneous change $\alpha_{i} \rightarrow \alpha \alpha_{i}$ and $q_{i} \rightarrow q q_{i}$ of all inverse masses and charges results in a change $E \rightarrow q^{4} \alpha^{-1} E$ and $r \rightarrow q^{-2} \alpha r$ of typical energies and distances. We thus can fix $q_{1}=1$, or $q_{1} q_{2}=1$, etc., and $\alpha_{1}+\alpha_{2}+\alpha_{3}=1$, or $\alpha_{1}+\alpha_{2}=1$, etc., at our convenience.

\section{B. Thresholds}

To be stable, a three-body system should lie below its lowest dissociation threshold, which corresponds to the more negative of the $(1,2)$ and $(1,3)$ binding energies. The separation between the two possible thresholds is given by

$$
\left(\alpha_{1}+\alpha_{2}\right)^{-1}\left(q_{1} q_{2}\right)^{2}=\left(\alpha_{1}+\alpha_{3}\right)^{-1}\left(q_{1} q_{3}\right)^{2},
$$

or, using (2),

$$
q_{2}^{2}\left(1-\alpha_{2}\right)=q_{3}^{2}\left(1-\alpha_{3}\right) .
$$

This is a straight line, hereafter referred to as $(T)$, passing through $A_{1}^{\prime}$, the symmetric of the upper vertex $A_{1}$ with respect to the lower side of the triangle, as seen in Fig. 3. Without loss of generality, one can assume $q_{2} \geq q_{3}$, so that $\alpha_{2} \geq \alpha_{3}$ at the threshold separation, which is thus slanted toward the right, as shown in Fig. 3.

\section{Star shape of the instability domains}

The star-shape property mentioned for unit charges remains valid for $q_{i} \neq 1$. It can be phrased as follows: a straight line issuing from $A_{2}$ or $A_{3}$ enters at most once the stability region until it reaches either the border of the triangle or the threshold separation $(T)$.

\section{Convexity}

The property of each instability region being convex also remains as the charges are no longer unity. The proofs given in Ref. [1] for the star shape and convexity properties are easily seen not to depend on $q_{i}=1$.

\section{E. Antisaturation}

If $q_{3}<q_{1}$, every point on the right of the threshold separation corresponds to a stable configuration. If, furthermore, $q_{3} \leq q_{2}<q_{1}$, every configuration is stable.

The proof easily results from the star-shape property. Let us, for instance, assume $q_{3}<q_{1}$. For $\alpha_{1}=\alpha_{3}=0$, i.e., for infinitely massive particles 1 and 3 , we have a pointlike $(1,3)$ atom with a residual negative charge, which at-

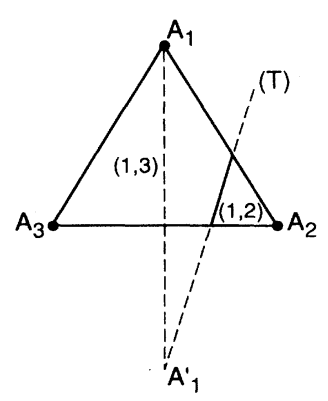

FIG. 3. Separation between the $[(1,2)-3]$ and $[(1,3)-2]$ thresholds. 
tracts a particle 2 with mass $\alpha_{2}^{-1}=1$. Thus the point $A_{2}$ belongs to the stability domain. Hence every point on the right of $(T)$ is stable. The same holds on the left side if $q_{2}<q_{1}$.

\section{F. Dependence on the charges}

If $q_{1}$ changes, the threshold separation $(T)$ does not move. One can rescale all charges so that $q_{1} q_{2}$ and $q_{1} q_{3}$, i.e., the threshold energies, remain constant, whereas $q_{2} q_{3}$ changes in the opposite direction. Thus increasing $q_{1}$ improves stability, and decreasing $q_{1}$ deteriorates it.

Consider now an increase of $q_{2}$, starting from a situation at the left of $(T)$, where $(1,2)$ is the lowest threshold. The line $(T)$ becomes more slanted, so we stay in the same sector. A rescaling can make $q_{1} q_{2}$ constant, i.e., the threshold energy constant. In the three-body Hamiltonian, the repulsion $q_{2} q_{3}$ also remains constant, while the attraction $q_{1} q_{3}$ decreases. We thus have less binding, and the parts of the frontier which lie at the left of $(T)$ move toward the right-hand side.

Similarly, a decrease of $q_{2}$ improves stability at the right of $(T)$, with, however, the warning that $(T)$ itself now moves to the left, so if one starts too close to $(T)$ one might end in the sector where $(1,3)$ is the lowest threshold.

Another case deals with a change $q_{2} \rightarrow q_{2}+\delta q_{2}$, when one starts from a point at the right of $(T)$. If $q_{2}$ increases, $(T)$ becomes more slanted toward the right, but we assume that $\delta q_{2}$ is small enough so that $(1,3)$ remains the lowest threshold. The corresponding threshold energy is constant. If we write the three-body Hamiltonian as

$$
H=h_{13}+\frac{\vec{p}_{2}^{2}}{2 m_{2}}+q_{2}\left[\frac{q_{3}}{r_{23}}-\frac{q_{1}}{r_{12}}\right] \text {, }
$$

necessarily

$$
\left\langle\Psi\left|\frac{q_{1}}{r_{12}}\right| \Psi\right\rangle>\left\langle\Psi\left|\frac{q_{3}}{r_{23}}\right| \Psi\right\rangle
$$

for a point inside the stability domain with wave function $\Psi$, otherwise $H$ cannot become smaller than $h_{13}$. This means that the first-order correction

$$
\delta E=\delta_{q 2}\left\langle\Psi\left|\frac{q_{3}}{r_{23}}-\frac{q_{1}}{r_{12}}\right| \Psi\right\rangle
$$

has a sign opposite to that of $\delta q_{2}$. If $q_{2}$ increases, stability is improved, and if $q_{2}$ decreases, it is deteriorated.

A similar study can be done with $q_{3}$ changing at fixed $q_{1}$ and $q_{2}$. An increase (decrease) of $q_{3}$ deteriorates (improves) binding at the right of $(T)$, and the reverse is true at the left of $(T)$.

To summarize, we have a simple monotonic behavior of the stability frontier in the triangular plot as the $q_{i}$ vary. Changing $q_{1}$ makes the domain wider or narrower. A change in $q_{2}$ or in $q_{3}$ modifies the slope of the line $(T)$ separating the thresholds, and the move of the frontier, if any, takes place in the same direction as $(T)$. An example is schematically pictured in Fig. 4.

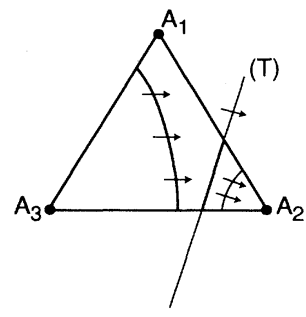

FIG. 4. Change of stability frontier as $q_{2}$ increases.

So far, we have used only qualitative arguments associated with the sign of the charges. One can also use the property of the lowest energy $E$ being a concave function of each $q_{i} q_{j}$, since these strengths enter the Hamiltonian linearly. One can also combine scaling with convexity, to improve convexity constraints [9]: $-(-E)^{1 / 2}$ is a concave function of each $q_{i} q_{j}$.

\section{THE BORN-OPPENHEIMER LIMIT}

Let us consider in this section the case where the masses of the positive charges are infinite, i.e., $\alpha_{1}=1$ and $\alpha_{2}=\alpha_{3}=0$, corresponding to the vertex $A_{1}$ of the triangle. One can fix $q_{1}=1$ and scan the plane $\left(q_{2}, q_{3}\right)$ to see where the system is stable. It is sufficient to calculate the ground-state energy $\epsilon_{0}(R)$ of the first particle as a function of the separation $R=r_{23}$ between the positive charges. Then one should look whether or not the effective potential

$$
V_{\mathrm{eff}}(R)=\epsilon_{0}(R)+\frac{q_{2} q_{3}}{R}
$$

remains above the threshold energy $-q_{2}^{2} / 2$. Solving the two-center Hamiltonian

$$
h=\frac{\vec{p}_{1}^{2}}{2}-\frac{q_{2}}{r_{2}}-\frac{q_{3}}{r_{3}}
$$

to compute $\epsilon_{0}(R)$ is a standard problem [10] (up to a scale factor, it depends only on the ratio $\left.q_{2} / q_{3}\right)$.

A detailed study has been performed recently by Hogreve [11]. Our investigations confirm his results, and supplement them, as we shall see, by an analysis of the behavior near $q_{3} \rightarrow 0$. The stability domain is shown in Fig. 5. Let us repeat that our definition of "stability" is kept as the one used in the finite-mass case, namely, that

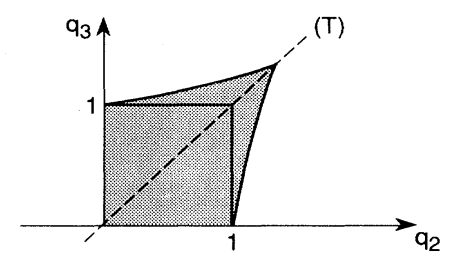

FIG. 5. Domain of stability in the Born-Oppenheimer limit where the like-sign charges $q_{2}$ and $q_{3}$ have infinite masses. 
the ground-state energy of the three-body system is lower than that of either of the two possible two-body systems.

The stability domain includes the square $\left(q_{2} \leq 1, q_{3} \leq 1\right)$, due to the antisaturation property mentioned in Sec. III. It extends mostly along the symmetry axis $q_{2}=q_{3}$, with an extreme peak near $q^{2}=q_{3} \simeq 1.24$ (1.236 67, according to Hogreve [11]).

A higher value $q_{2}=q_{3} \simeq 1.44$ is sometimes quoted. It corresponds to the limit for metastability. For $q_{2}=q_{3}$ and $1.24<q_{2}<1.44$, the effective potential has a local minimum in which the heavy particles are trapped, but this minimum lies above the dissociation threshold.

When $q_{3}$ is small, one can use perturbation theory to show that the frontier behaves as

$$
q_{2}-1 \simeq 18 \frac{q_{3}}{\left(-\ln q_{3}\right)^{3}},
$$

and thus starts vertically, as pictured in Fig. 5 .

The proof is the following. At zeroth order in $q_{3}$, we have a $(1,2)$ atom, with energy $-q_{2}^{2} / 2$ and wave function

$$
\pi^{-1 / 2} q_{2}^{3 / 2} \exp \left(-q_{2} r\right)=\frac{1}{\sqrt{4 \pi}} \frac{u(r)}{r},
$$

with $\vec{r}=\vec{r}_{1}-\vec{r}_{2}$, and $r=|\vec{r}|$. At first order, the charge $q_{3}$, located at $\vec{r}_{3}=\vec{r}_{2}+\vec{R}$, has an energy

$$
\delta E_{1}=q_{3}\left[\frac{q_{2}}{R}-\left\langle\frac{1}{|\vec{r}-\vec{R}|}\right\rangle\right] \text {. }
$$

The expectation value is computed using the above wave function, with the well-known result

$$
\langle\rangle=\frac{1}{R} \int_{0}^{R} u^{2}(r) d r+\int_{R}^{\infty} \frac{u^{2}(r)}{r} d r .
$$

Thus

$$
\left\langle>\leq \frac{1}{R} \int_{0}^{\infty} u^{2}(r) d r=\frac{1}{R},\right.
$$

and $\delta E_{1} \geq q_{3}\left(q_{2}-1\right) / R$ is positive if $q_{2}>1$. Thus the stability frontier ends at $\left(q_{2}=1, q_{3}=0\right)$.

To make more precise the behavior of the frontier near the end point, we push the perturbative expansion up to second order, and write the effective potential between the heavy charges as

$$
V_{\mathrm{eff}}\left(q_{2}, q_{3}, R\right)=-\frac{q_{2}^{2}}{2}+\delta E_{1}+\delta E_{2}
$$

with an explicit computation of $\delta E_{1}$ of Eq. (13) as

$$
\delta E_{1}=q_{3}\left[\frac{q_{2}}{R}-\frac{f\left(q_{2} R\right)}{R}\right],
$$

where the form factor is

$$
f(x)=1-(1+x) \exp (-x) .
$$

We now assume, and will shortly check, that if there is any binding for small $q_{3}$ it occurs at large separation $R$. Then the second-order effect is calculated in the approximation of a constant electric field $q_{3} / R^{2}$ acting on the atom. The Stark effect is well known and reads

$$
\delta E_{2}=-\frac{9 q_{3}^{2}}{4 R^{4} q_{2}^{4}} .
$$

If one rewrites (16) as

$$
V_{\mathrm{eff}}=-\frac{q_{2}^{2}}{2}+\frac{F(R)}{R}
$$

the stability frontier corresponds to $F(R)=0$ and $F^{\prime}(R)=0$, which in the limit $q_{2} \rightarrow 1$ and $q_{3} \rightarrow 0$ reads

$$
\begin{aligned}
& \left(q_{2}-1\right)+(1+R) \exp (-2 R)-\frac{9 q_{3}}{4 R^{3}}=0, \\
& -(2 R+1) \exp (-2 R)+\frac{27 q_{3}}{4 R^{4}}=0 .
\end{aligned}
$$

Disregarding the spurious small- $R$ solution due to approximation (19), we see that the second equation of (21) shows that $R$ is, indeed, very large, $R \sim\left|\ln q_{3}\right| / 2$. A simple elimination leads to the desired result (11).

\section{STABILITY OF NEARLY SYMMETRIC SYSTEMS}

In Ref. [1], we considered the case of unit charges. Many accurate calculations exist for symmetric systems, with inverse masses proportional to $\alpha_{1}, \alpha_{2}=\left(1-\alpha_{1}\right) / 2$, and $\alpha_{3}=\alpha_{2}$. If their binding energy is written as

$$
E\left(\alpha_{1}\right)=\left[1+g\left(\alpha_{1}\right)\right] E_{\mathrm{th}},
$$

where $g\left(\alpha_{1}\right)$ denotes the fraction of binding below the threshold, one can set a lower limit to the extension of the stability domain for a given $\alpha_{1}[1]$,

$$
\delta \geq \frac{2}{\sqrt{3}} \frac{g\left(\alpha_{1}\right)}{1+g\left(\alpha_{1}\right)}\left(1+\alpha_{1}\right) .
$$

The width $\delta$, defined in Fig. 2, is the maximal allowed value for $2\left|\alpha_{2}-\alpha_{3}\right| / \sqrt{3}$

The proof is very simple [1]. The threshold of the system $\left(\alpha_{1}, \alpha_{2}, \alpha_{3}\right)$ is exactly known, and its energy is bounded by rewriting

$$
\begin{aligned}
H\left(\alpha_{1}, \alpha_{2}, \alpha_{3}\right)= & H\left[\alpha_{1}, \frac{\alpha_{2}+\alpha_{3}}{2}, \frac{\alpha_{2}+\alpha_{3}}{2}\right] \\
& +\frac{\alpha_{2}-\alpha_{3}}{4}\left(\vec{p}_{3}^{2}-\vec{p}_{2}^{2}\right) .
\end{aligned}
$$

This ensures that

$$
E\left(\alpha_{1}, \alpha_{2}, \alpha_{3}\right) \leq E\left[\alpha_{1}, \frac{\alpha_{2}+\alpha_{3}}{2}, \frac{\alpha_{2}+\alpha_{3}}{2}\right),
$$

since the antisymmetric term in (24) necessarily lowers the ground state, a simple consequence of the variational principle.

The same reasoning can be applied to the strengths $s_{1}=q_{2} q_{3}, s_{2}=q_{3} q_{1}$, and $s_{3}=q_{1} q_{2}$. For a given symmetric mass distribution $\left(\alpha_{2}=\alpha_{3}\right)$, one has

$$
E\left(s_{1}, s_{2}, s_{3}\right) \leq E\left[s_{1}, \frac{s_{2}+s_{3}}{2}, \frac{s_{2}+s_{3}}{2}\right] \text {. }
$$




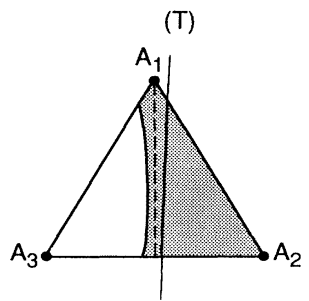

FIG. 6. For charges $q_{1}=1, q_{2}=1.02$, and $q_{3}=0.98$, the stability domain extends at least from $A_{2}$ to the median axis of the triangle.

If, for instance,

$$
s_{1}=1, s_{2}=1-x, s_{3}=1+x,
$$

stability is guaranteed at least for

$$
(1+x)^{2} \leq 1+g \text {. }
$$

One can solve (27) for the charges, rescale to $q_{1}=1$, and then say that (28) means stability for configurations corresponding to parameters

$$
\alpha_{i}=\left(\alpha_{1}, \frac{1-\alpha_{1}}{2}, \frac{1-\alpha_{1}}{2}\right), \quad q_{i}=\left(1, \frac{1}{1-x}, \frac{1}{1+x}\right) \text {. }
$$

When $\alpha_{1}$ varies, the minimum of $g$ is between $4 \%$ and $5 \%$. Then, if $x \leq 0.02$, we are sure that the symmetry axis of the triangle entirely belongs to the stability domain, as shown in Fig. 6.

\section{STABILITY DOMAIN FOR GIVEN CHARGES}

We have a variety of scenarios for the stability region in the triangle of normalized inverse masses. We first fix $q_{1}=1$, and consider the case of equal charges $q_{2}=q_{3}$. For $q_{2}<1$, every configuration is stable.

For $q_{2}=1$, we have the central band of Fig. 2. There is a sudden change as $q_{2}$ becomes 1 , when one compares in the stability plot the three-body energy $E^{(3)}$ with the two-body energy $E^{(2)}$, but this does not contradict the property of $E^{(3)}$ and $E^{(2)}$ being both continuous.

As $q_{2}$ further increases, the band becomes narrower, and then presumably breaks slightly below the center of the triangle. Indeed, for $q_{2}=1$, the relative excess energy $g\left(\alpha_{1}\right)$ of Eq. (22) is maximal for $\alpha_{1}=1\left(\mathrm{H}_{2}{ }^{+}\right.$with $\left.m_{p}=\infty\right)$, but is not minimal for $\alpha_{1}=0$. Near $\alpha_{1}=0, \mathrm{H}^{-}$ becomes less stable as the proton mass becomes finite, as seen from existing accurate calculations [12]. The minimum of $g\left(\alpha_{1}\right)$ occurs around $\alpha_{1} \simeq 0.2$ (see, for instance, the plot in Fig. 2 of Ref. [1]), intermediate between $\mathrm{Ps}^{-}$and $\mathrm{H}^{-}$. We thus expect the breaking as $q_{2}$ increases to take place near this $\alpha_{1} \simeq 0.2$.

For values of $q_{2}$ just above this breaking, two islands of stability remain, one in the Born-Oppenheimer region $A_{1}$ $\left(\mathrm{H}_{2}^{+}\right)$, and another at the middle of $A_{2} A_{3}\left(\mathrm{H}^{-}\right)$. This is illustrated in Fig. 7.
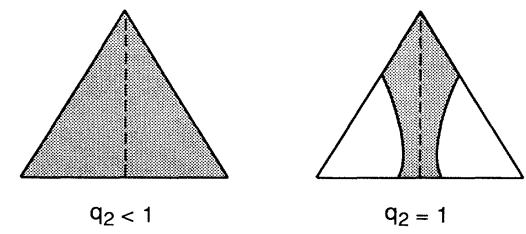

$q_{2}=1$

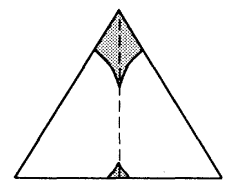

$q_{2} \simeq 1.09$

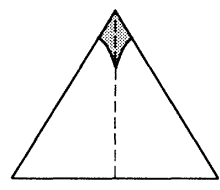

$1.1<q_{2}<1.24$

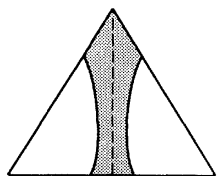

$q_{2} \gtrsim 1$

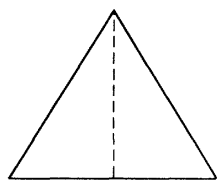

$q_{2}>1.24$
FIG. 7. Shape of the stability region for $q_{1}=1$ and increasing values of the equal charges $q_{2}=q_{3}$.

As shown by Baker et al. [13], stability is lost for $\mathbf{H}^{-}$ near $q_{2}=q_{3}=1.0975$, while the study of Hogreve [11] and the results of Sec. IV show that $\mathrm{H}_{2}{ }^{+}$survives up to $q_{2}=q_{3}=1.24$. From these results it is easy to see by concavity considerations that stability is completely lost for $q_{2}=q_{3}>1.24$ for all masses. The fact that the stability region splits into two separate domains for $q_{2} \simeq 1.09$ follows from the work of Pekeris [14] on recoil corrections in $Z e^{-} e^{-}$.

Let us now turn to the case where $q_{3}<q_{2}$. The ratio $q_{2} / q_{3}$ uniquely determines the slope of $(T)$, the line separating the thresholds, according to Eq. (5). Some examples are given in Fig. 8, and commented upon below.

For $q_{3}<q_{2}<1$, every configuration is stable. For $q_{3}<q_{2}=1$, one should observe a sudden change, as some configurations near $A_{3}$ become unstable.

The picture is qualitatively similar for $q_{3}<1<q_{2}$, with stability at the right-hand side of $(T)$, and by continuity somewhat at the left of $(T)$. If, for instance, $q_{3}=0.95$

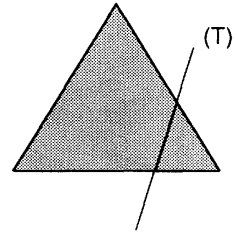

$q_{3}<q_{2}<1$

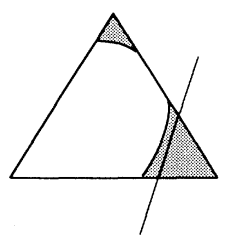

$\mathrm{q}_{3}<\mathrm{q}_{2}=1$ $q_{3}$ small

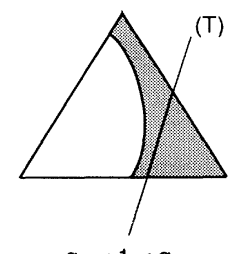

$\mathrm{q}_{3}<1<\mathrm{q}_{2}$ $q_{3} / q_{2}$ not too small

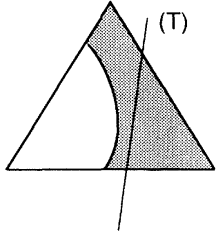

$q_{3}<q_{2}=1$

$\mathrm{q}_{3}$ not too small

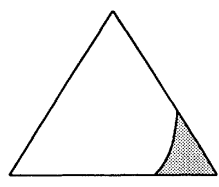

$q_{3}<1<q_{2}$

$q_{3} / q_{2}$ small
FIG. 8. Shape of the stability region for $q_{1}=1$ and various values of the positive charges $q_{3}<q_{2}$. 
and $q_{2}=1.05$, corresponding to the lower middle case, the stability domain includes the Born-Oppenheimer point $A_{1}$, while for, say, $q_{3}=0.5$ and $q_{2}=1.2$, roughly the situation of the lower right case, we are restricted to a limited area near $\Lambda_{2}$. This will be studied into more detail in Ref. [14].

For $q_{3}=1<q_{2}$, we observe another sudden change: some configurations near $A_{2}$ become unstable. For $1<q_{3}<q_{2}$, the stability region includes at most a band around $(T)$, which can break into two or three islands, and eventually disappear. For a moderate ratio $q_{2} / q_{3}$, we have a deformed version of the situation depicted for $q_{2}=q_{3}$ in Fig. 7. The situation becomes more delicate for large values of $q_{2} / q_{3}$ : here, one does not expect any stability, except perhaps for $q_{3}$ very close to 1 . A study of the large $-q_{2} / q_{3}$ limit will be given in [7].

\section{STABILITY DOMAIN FOR GIVEN MASSES}

We now consider the stability domain for given masses in the $\left(q_{2}, q_{3}\right)$ plane. In this section the concavity of $-\sqrt{-E}$ with respect to $q_{2}$ for fixed $q_{3}$ and to $q_{3}$ for fixed $q_{2}$ is essential. From it we can prove that the two arcs forming the border are intersected only once by horizontal or vertical lines. The normalization is again $q_{1}=1$ in this section. We note that the square $\left(q_{2}<1, q_{3}<1\right)$ always corresponds to stability. The nontrivial question is thus to find some extension outside this square. In the case where $m_{2}=m_{3}=\infty$, shown in Fig. 4, we have seen that this extension essentially consists of a spike along the symmetry axis.

Consider first equal but finite masses $m_{2}=m_{3}$. For very small $q_{3}$, and $q_{2} \geq 1$, the system is unstable. The frontier actually departs from the square $\left(q_{2}<1, q_{3}<1\right)$ at some finite value of $q_{3}$, as shown in Fig. 9(a). There is an extremum on the symmetry axis $q_{2}=q_{3}$, below the value $q_{2}=1.24$ obtained for infinite masses. If, indeed, we look at the limit $m_{2}=m_{3} \rightarrow \infty$, we can approximate the Born-Oppenheimer potential by a harmonic-oscillator
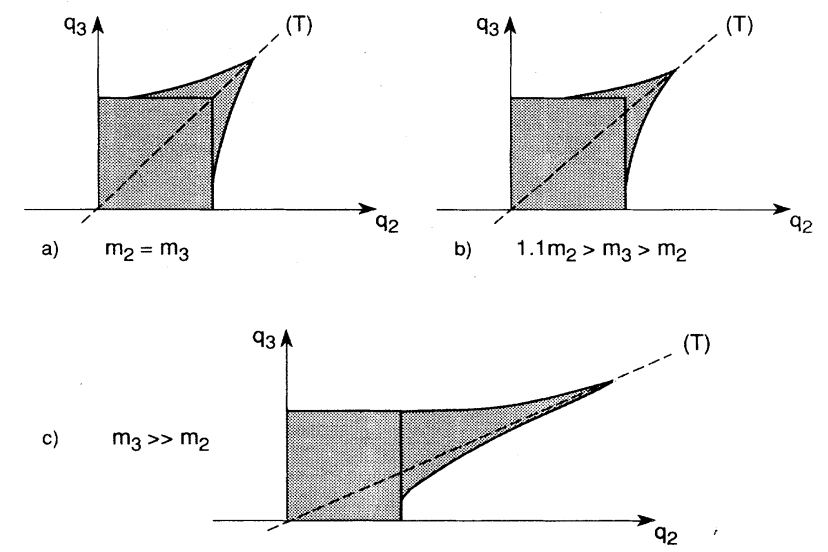

FIG. 9. Shape of the stability region in the $\left(q_{2}, q_{3}\right)$ plane for $q_{1}=1$ and various values of the masses. well near its minimum, resulting in a binding energy of the type

$$
E^{(3)} \simeq A+\frac{B}{\sqrt{m_{2}}}, \quad B>0 .
$$

Thus $E^{(3)}$ varies quite sharply, while the threshold energy $E^{(2)}$ is a smooth function of $m_{2}$. Stability becomes harder when $m_{2}$ is finite.

Let us now assume an asymmetric $m_{2}<m_{3}$. In the $\left(q_{2}, q_{3}\right)$ plot, the separation $(T)$ between the thresholds is also a straight line, given by Eq. (5).

Consider first the case where the masses are such that we have stability for unit charges. The picture in the $\left(q_{2}, q_{3}\right)$ plot is then qualitatively similar to the previous ones. An illustration is given in Fig. 9(b). Stability is ensured in the square $\left(q_{2}<1, q_{3}<1\right)$ and in the triangle limited by $q_{3}=1$ and $(T)$, and extends somehow outside.

We can finally imagine a larger asymmetry between $m_{2}$ and $m_{3}$, so that the point $q_{2}=q_{3}=1$ does not correspond to stability. $(T)$ can now be extremely slanted. This means stability for $q_{1}=1, q_{3} \simeq 1$, and very large $q_{2}$. This is illustrated in Fig. 9(c).

\section{OUTLOOK}

In this paper, we have sketched the possible shapes of the stability domain for various values of the masses and charges. The main lesson is the following. We always obtain stability when the like-sign charges $q_{2}$ and $q_{3}$ are smaller than $q_{1}$, whatever masses are involved. If, say, only $q_{3}<q_{1}$, we have a rather obvious stability when both masses $m_{1}$ and $m_{3}$ are relatively heavy, so that $(1,3)$ acts as a localized attractive center for particle 2 ; more precisely, $q_{3}<q_{1}$ implies stability at least as long as $(1,3)$ remains the lowest threshold. It is much less obvious that one can obtain stability with both $q_{2} \geq q_{1}$ and $q_{3} \geq q_{1}$, and indeed this usually does not lead to a stable compound. The remarkable exceptions correspond to situations where the thresholds energies are nearly equal. This is seen as a band around the threshold separation $(T)$ in the triangular plot of inverse masses at fixed charges, or as a spike along $(T)$ in the $\left(q_{2}, q_{3}\right)$ plot at fixed masses and fixed $q_{1}=1$.

In other words, there are only two ways for a threecharge system to be stable: the trivial way $\left(q_{2}<1\right.$ and/or $q_{3}<1$ ), corresponding to an obvious excess of attraction; otherwise the two threshold energies being nearly equal. In this case the wave function contains a coherent superposition of [1-2]-3 and [1-3]-2 cluster structures. This mechanism is well known in few-body physics. In particular, experts in variational calculations are aware that the nearest thresholds should be introduced in the trial wave function, and that competing thresholds tend to generate attraction.

The present work could be extended in several directions. Let us list a few of these.

(i) The behavior of the frontier near end points could be further elaborated.

(ii) Many variational methods have been developed for such Coulomb systems. They could be applied to esti- 
mate quantitatively the location of the frontier, with a high accuracy.

(iii) One would like to know the envelope of the stability curves in the $\left(q_{2}, q_{3}\right)$ plane when one varies the masses. There is, in the $\left(q_{2}, q_{3}\right)$ plane, a domain where the threebody system is unstable for all masses. At present, very little is known about this domain.

(iv) A similar study could be done with other potentials. For instance, with power-law potentials $V \propto r_{i j}^{\beta}$, we have similar scaling laws and virial relations as for the $\beta=-1$ case considered here, and thus the same number of variables in the stability plots. The convexity arguments developed in [1] and in the present paper hold in fact for any potential that does not depend on the masses.

(v) Generalization to four particles seems technically difficult, but rather tempting. One could guess one would again obtain stability in two cases: an obvious excess of attraction, i.e., $\sum q_{i} q_{j}<0(i \neq j)$, and also a conspiration of thresholds, i.e., with an appropriate numbering

$$
\frac{\left(q_{1} q_{3}\right)^{2}}{\alpha_{1}+\alpha_{3}}+\frac{\left(q_{2} q_{4}\right)^{2}}{\alpha_{2}+\alpha_{4}} \simeq \frac{\left(q_{1} q_{4}\right)^{2}}{\alpha_{1}+\alpha_{4}}+\frac{\left(q_{2} q_{3}\right)^{2}}{\alpha_{2}+\alpha_{3}} .
$$

(vi) One could then look whether the stability conditions are likely to be fulfilled in other systems. For instance, it is an open problem in hadron physics whether or not stable multiquark systems exist [15].

\section{ACKNOWLEDGMENTS}

We thank M. B. Ruskai and J. Stubbe for stimulating discussions. One of us (T.T.W.) benefited from the warm hospitality of the theory division at CERN, and his work was supported in part by the U.S. Department of Energy under Grant No. DE-FG02-84-ER40158.
[1] A. Martin, J.-M. Richard, and T. T. Wu, Phys. Rev. A 43, 3697 (1992)

[2] A. H. Frolov and D. M. Bishop, Phys. Rev. A 45, 6236 (1992).

[3] A. V. Gur'yanov and T. K. Rebane, Zh. Eksp. Teor. Fiz. 98, 65 (1990) [Sov. Phys. JETP 71, 34 (1991)].

[4] E. A. G. Armour and W. Byers Brown, Acc. Chem. Res. 26, 168 (1993).

[5] Z. Chen and L. Spruch, Phys. Rev. A 42, 133 (1990).

[6] S. S. Gershtein and L. I. Ponomarev, in Muon Physics, edited by V. W. Hugues and C. S. Wu (Academic, New York, 1975), Vol. III.

[7] A. Martin, J.-M. Richard, and T. T. Wu (unpublished).

[8] R. N. Hill, J. Math. Phys. 18, 2316 (1977).
[9] W. Thirring, Quantum Mechanics of Atoms and Molecules, A Course in Mathematical Physics Vol. 3 (SpringerVerlag, New York, 1983).

[10] See, for instance, H. Wind, J. Chem. Phys. 42, 2371 (1965); 43, 2956 (1965), and references therein to earlier works.

[11] H. Hogreve, J. Chem. Phys. 98, 5579 (1993).

[12] J. F. Hart and G. Hertzberg, Phys. Rev. 106, 79 (1957).

[13] J. D. Baker, D. E. Freund, R. N. Hill, and J. D. Morgan, Phys. Rev. A 41, 1247 (1990).

[14] C. L. Pekeris, Phys. Rev. 112, 1649 (1958).

[15] J.-M. Richard, in Quark Cluster Dynamics, Proceedings of the Bad Honnef Workshop, 1992, edited by K. Goeke, P. Kroll, and H. R. Petry (Springer-Verlag, Berlin, 1993). 